\title{
Prototipo de bioingenieria para el traslado y pesaje de pacientes hospitalizados con sobrepeso o movilidad limitada
}

\author{
Ortega-Loayza Daniel'; Cisneros-Morales Cristián²; Tabuchi-Yaqui Toshiro²; \\ Arteaga-Chávez Luis²; Mayorca-Carmelo Carmen ${ }^{3}$; Vaiz-Bonifaz Rosa ${ }^{3}$, Bolaños-Hil Hilda ${ }^{3}$; \\ Salazar-García María del Carmen ${ }^{4}$; Farro-Peña Gianina ${ }^{5}$
}

\section{RESUMEN}

Objetivo: diseñar, fabricar y realizar pruebas de un prototipo de sistema electromecánico para mejorar la atención a pacientes hospitalizados, con sobrepeso o problemas de movilidad, en los servicios de enfermería. Material y métodos: estudio de investigación experimental, como base de prueba, realizado por los profesores de las facultades de Enfermería de la Universidad Peruana Cayetano Heredia y Ingeniería Mecánica de la Universidad Nacional de Ingeniería. Se partió de la realidad de los servicios de un hospital del Ministerio de Salud-Lima. Resultados: se presentó el prototipo integrando la camilla recta, la balanza electrónica y el sistema de comunicación vía bluetooth, cuyas funcionalidades (lectura de pesos, uso y manejo del sistema integrado) fueron explicadas al personal de la Facultad de Enfermería para su posterior validación con los pacientes. Conclusiones: el prototipo de sistema bioingenieril contribuye a mejorar la calidad de atención en el pesaje y en el traslado de manera segura y cómoda de pacientes hospitalizados y evitar lesiones físicas al personal de salud.

Palabras clave: bioingeniería, mecánica, electrónica, ergonomía, transferencia de pacientes, enfermería. Perú (Fuente DeCs BIREME).

\section{Prototype of bioengineering for the transfer and weighing inpatient overweight or limited mobility}

\begin{abstract}
Objetive: Design, manufacture and testing of a prototype of electromechanical system to improve care to hospitalized patients, overweight or problems of mobility in nursing services. Material and Methods: Study of experimental research, as a basis for testing, conducted by professors from the faculties of Nursing of the Cayetano Heredia Peruvian University and Mechanical Engineering of the National University of Engineering. It departed from the reality of the services of a hospital of the Ministry of health-Lima. Results: Presented the prototype integrating the stretcher straight, the electronic scale and the communication system via bluetooth, whose capabilities (weights, use and management of the integrated reading) were explained to the staff of the school of nursing for further validation with patients. Conclusions. The prototype bioingenieril system contributes to improve the quality of care at the weigh-in and in the transfer of safe and comfortable way of hospitalized patients and prevent injury to health personnel.
\end{abstract}

Key words: Bioengineering, mechanics, electronics, ergonomics, transfer of patients, nursing. Peru (Source DeCs BIREME).

Doctor en Administración. Docente de la Universidad Nacional de Ingeniería. Lima,Perú

Bachiller. Universidad Nacional de Ingeniería. Lima,Perú

Magister en enfermería. Docente de la Universidad Peruana Cayetano Heredia. Lima,Perú.

Licenciada en enfermería. Docente de la Universidad Peruana Cayetano Heredia. Lima,Perú.

Magister en Salud Pública. Docente de la Universidad Peruana Cayetano Heredia. Lima,Perú. 


\section{INTRODUCCIÓN}

El presente es un proyecto multidisciplinario y sectorial realizado con el fin de revelar la aplicación de la ingeniería en la medicina, lo que da lugar a la Bioingeniería o Ingeniería Biomédica, mediante la integración de varias ramas de estudio como las ciencias generales (Biología, Fisiología, Química, Física, Matemáticas, Electrónica e Informática), las ciencias básicas interdisciplinarias (Biofísica, Bioma temática, Bioquímica) y las disciplinas componentes (Bioinstrumentación, Bioelectrónica, Biomecánica, Biocibernética, Biónica, Bioinformática, Procesamiento de señales biológicas) (1). Esta tecnología es en la actualidad muy importante debido a sus grandes avances en equipos, prótesis, dispositivos, y herramientas para el tratamiento y diagnóstico de enfermedades (2). La bioingeniería se aplica en el desarrollo de equipos que apoyen y beneficien al personal de la salud y a los pacientes hospitalizados, disciplina en la que nos enfocamos $(3,14)$.

Debido a la gran necesidad de tecnología en el área de la medicina la elaboración de equipos biomédicos se ha convertido en una parte fundamental en el tratamiento y atención de pacientes, y no es una novedad, puesto que desde el siglo XIX se vienen usando diversos equipos que con el avance del tiempo se han ido mejorando y creando nuevas tecnologías que ayudan de manera importante en este campo, tanto para laboratorios como para atención y cuidado del mismo paciente. En la actualidad es una herramienta vital para diagnosticar de forma efectiva, dar una rehabilitación o un buen tratamiento. Entre estos equipos se puede mencionar a la balanza, grúas, sillas y camas entre otros (4).

El Centro Nacional de Alimentación y Nutrición del Instituto Nacional de Salud reporta que el sobrepeso en la población peruana bordea el 35\%, mientras que la obesidad el 17\% (5). Adicionalmente, el cuidado enfermero a los pacientes hospitalizados toma como punto de referencia el peso del paciente, sin embargo, en el Ministerio de Salud (MINSA) y en el Seguro Social del Perú (EsSalud) solo tienen básculas de pie que requieren que el paciente se encuentre en bipedestación para poder realizar el control de peso respectivo. Usualmente, el equipo de enfermería que no cuenta con la información del peso del paciente para poder brindar la terapéutica farmacológica exacta y reponer los líquidos necesarios, tiende a aproximar este peso de acuerdo a la masa, cálculo que no es exacto si se desea brindar cuidados de calidad.

Actualmente, en un servicio de 25 camas el $60 \%$ de los pacientes está postrado y el $30 \%$ son obesos. La compra de camas eléctricas involucraría un gasto de 350000 o 500000 nuevos soles, solo en el servicio de un solo hospital. En contraposición, si consideramos que a escala nacional estamos por sobre las 25000 camas hospitalarias, 15000 pacientes se podrían beneficiar. La creación de tecnología nacional de acuerdo con nuestros requerimientos podría llevar al país a ahorrar millones de nuevos soles, que pudieran invertirse en la mejora de la calidad de atención.

Por lo tanto, el objetivo de esta investigación es diseñar, fabricar y validar un prototipo de sistema electrónico-mecánico para pesaje y la atención en el traslado de pacientes con sobrepeso o movilidad limitada, con el fin de brindar la terapéutica exacta, y de calidad, y prevenir lesiones físicas al paciente y al personal de salud.

En los hospitales y centros de atención médica es común encontrar pacientes con limitaciones de movilidad y sobrepeso. Esta situación se ha confirmado a través del análisis de las encuestas aplicadas en el proceso de investigación (6). El problema es que por su estado de salud muchos pacientes no pueden ser traslados de una cama a otra o a una camilla, y viceversa. Esto tiene dos serios inconvenientes: primero, que el paciente puede sufrir algún daño mientras se realizan las maniobras para trasladarlo; segundo, con el tiempo el personal de enfermería sufre problemas lumbares debido al esfuerzo físico realizado al trasladar a los pacientes.

La población adulta en el Perú ascendió a 10\% en el año 2000 y se estima que ascenderá a $16,6 \%$ en 2030 . La prevalencia de enfermedades crónicas degenerativas y el envejecimiento de la población también están en subida. En los hospitales, la edad de los pacientes supera los 80 años y el peso oscila entre 50 y 90 kilos. Algunos pacientes, sometidos a cirugía y que están bajo efectos de la anestesia, no pueden pesarse ni trasladarse, lo cual constituye un reto para el personal de salud. Al estar hospitalizados, estos pacientes presentan una serie de requerimientos que no pueden satisfacerse debido a la dificultad para medir el peso corporal por la postración y el traslado. No se cuentan con dispositivos o equipos que permitan el pesaje exacto y el traslado apropiado de estos pacientes $(7,15)$.

El uso de la mecánica corporal no es suficiente para vencer la resistencia corporal, por lo que resulta necesario que varias personas colaboren para movilizar a un paciente, y en algunos servicios o algunos horarios no se cuenta con la cantidad de personas para mover a un paciente obeso. El esfuerzo máximo de una mujer no debe 
exceder los 20 kilos. Para el varón el límite es 50 kilos (8). Así lo demuestran investigaciones realizadas en varios países en las que la aparición de trastornos músculoesqueléticos en trabajadores de enfermería presenta tasas de prevalencia por encima del 80\% (9).

Los dispositivos disponibles en el mercado son: la báscula multifunción con pasamanos o para silla de ruedas 664 676 - 684 - 952 creadas en España (10), requiere que el paciente se encuentre en bipedestación o sentado en una silla de ruedas, no puede usarse en pacientes postrados. Opcionalmente, este dispositivo permite colocar un tallímetro para calcular el índice de masa corporal (7). La báscula digital para cama o diálisis con mesa de transporte permite pesar a pacientes postrados, con un sistema de elevadores integrados de cuatro plataformas debajo de las ruedas de la cama. Esta báscula de cama para uso médico WPT/4B (11) tiene un precio en el mercado de 2140 euros. La báscula de cama con rampas para uso médico WPT/8B (3), muy similar a la anterior tiene un precio de 2140 euros. La cama eléctrica (2), que incluye la báscula metabólica disponible en algunos ambientes de lujo de clínicas de Lima, cuesta aproximadamente entre 25000 y 35000 dólares americanos. Algunas investigaciones en salud mencionan que cuando se requiere el control de peso de pacientes postrados se ha tenido que cargarlos y colocarlos en una camilla especial para pesarlo en una balanza tipo romana (4), esto podría ocasionar problemas biofísicos en el personal, y no todos los pacientes postrados pueden asumir ciertas posturas corporales. No existe en el medio, antecedentes de diseño de equipo o dispositivo para el traslado de pacientes postrados de cama a camilla o viceversa. Los equipos disponibles en el mercado para efectuar este tipo de funciones son importados y costosos, por lo que son poco asequibles para muchos centros de salud. Además, solo sirven para levantar al paciente que está sentado.

La propuesta presentada soluciona estos requerimientos levantando al paciente completamente echado (en el caso de pacientes con sobrepeso o movilidad limitada); además de controlar el peso continuamente mediante un sistema de comunicación electrónica. En el mercado no se encuentra el prototipo de equipo que este proyecto propone realizar. Esta línea de investigación podría abrir camino para la empresa peruana en el diseño de equipos y dispositivos acordes a las demandas de salud a bajo costo y con elevada aceptabilidad, preservando la seguridad del paciente y la comodidad de su atención. Adicionalmente, se favorece el vínculo desde el campo de la salud con el de las ingenierías para el desarrollo de innovación tecnológica propia del país.
La bioingeniería es una rama joven de la ingeniería en la cual los principios y las herramientas de la ingeniería, la ciencia y la tecnología se aplican a la resolución de problemas que se presentan en biología y, especialmente, en la salud (12). La IEEE (13) define la bioingeniería como: La ciencia que estudia y busca la aplicación de principios y métodos de las ciencias exactas, en general, y de la ingeniería, en particular, a la solución de problemas de las ciencias biológicas y médicas.

El cuidado o la atención a los pacientes se centra en resolver sus problemas de salud de diversa complejidad; la demanda en el personal es el empleo de tecnología blanda (atención directa) y la tecnología dura (diversos equipos mecánicos, eléctricos, etcétera). Esta tecnología es un medio para brindar el cuidado; en la actualidad ante un paciente postrado o con movilidad limitada por enfermedad, o en posoperatorio inmediato, es necesario obtener el peso exacto; el traslado de camilla a cama se constituye en riesgo para la seguridad de los pacientes, sobre todo en pacientes con sobrepeso u obesidad, y la posibilidad de lesiones músculoesqueléticas en el personal a cargo, a pesar de emplear la mecánica corporal, esta es una situación percibida por las enfermeras que laboran en hospitales del Ministerio de Salud, de la seguridad social y otras instituciones, según sondeo de opinión realizada últimamente.

\section{MATERIAL Y MÉTODOS}

Estudio de investigación experimental, como base de prueba, realizado por profesores de la Facultad de Enfermería de la Universidad Peruana Cayetano Heredia (UPCH) y la Facultad de Ingeniería Mecánica de la Universidad Nacional de Ingeniería (UNI). Se partió de la realidad de los servicios de un hospital del Ministerio de Salud (MINSA), Lima. Se desarrolló usando la metodología de la ingeniería basada en la combinación del diseño secuencial y múltiples alternativas aplicadas al diseño, la logística y la construcción del método conocido como Lean Project Delivery System, que considera cinco fases y 13 módulos para la gestión de un proyecto.

Se propuso una solución con el prototipo de sistema electrónico-mecánico adecuadamente diseñado y fabricado, probado y validado, el cual facilitará brindar la terapéutica exacta y un adecuado balance hídrico al paciente, teniendo como referencia el peso. Además de contar con un dispositivo que permita el desplazamiento del paciente con menor esfuerzo físico del personal reduciendo los costos de atención. 
La propuesta incluye dispositivos para traspaso (elevación y desplazamiento) de pacientes con problemas de movilidad y/o sobrepeso de la cama clínica a la camilla, una o varias estructuras de pesaje de pacientes y un sistema de comunicación inalámbrica para transferir hacia un Smartphone los datos de peso registrados por el sistema de pesaje electrónico. Se formula una nueva línea de investigación, denominada sistematización y electromecanización en la calidad de atención a pacientes hospitalizados con limitaciones, cuyos resultados aportaron conocimientos a los centros de investigación de ambas universidades (UPCH - UNI).
Sistema integrado: una vez que se conectaron todos los circuitos electrónicos, se repitieron las mismas pruebas y se obtuvieron los mismos resultados satisfactorios.

El sistema se compone de una microcomputadora central, basada en el microcontrolador ATmega328P con módulos de conversión de señal análoga a digital de 10 bits de resolución, comunicación SPI, comunicación RS232, y procesador de $8 \mathrm{MHz}$. Adicionalmente, se le ha incluido un circuito de carga para batería recargable de ión-litio, un módulo de memoria externa expandible micro SD y un módulo de comunicación inalámbrica bluetooth.

Figura 1. Diagrama de bloques del sistema integrado

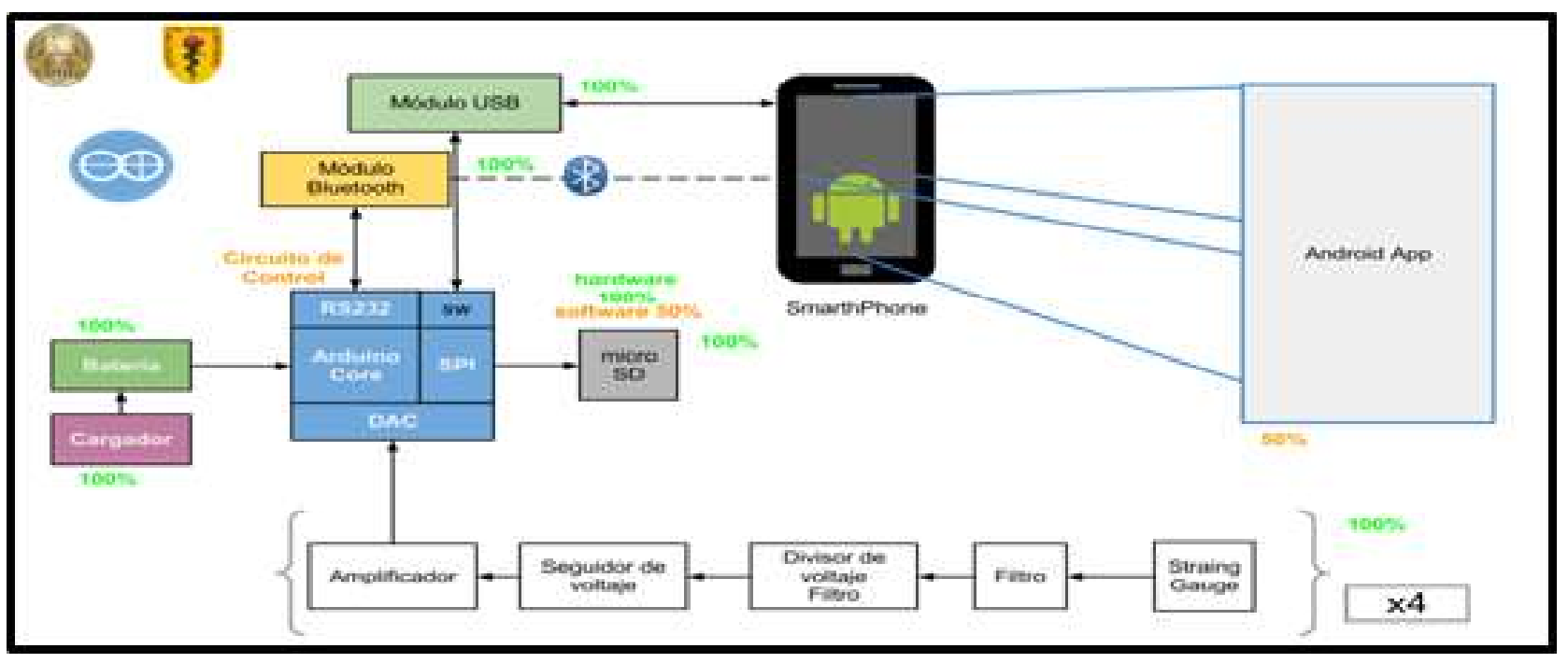

\section{RESULTADOS Y DISCUSIÓN}

1. De la evaluación con las personas involucradas se concluyó que era necesaria una camilla integrada al prototipo, para ello, el equipo de investigación de la UNI tomó las medidas necesarias para modificar el prototipo y proceder a una nueva entrega.

Luego, se presenta el prototipo integrando la camilla plana sugerida, y la balanza electrónica con todos los componentes necesarios implementados por el equipo de investigación de la UNI.

2. Tras las pruebas realizadas, el equipo de investigación de la UNI formula correcciones al prototipo grúa. El equipo de investigación de la UNI plantea correcciones a las deficiencias observadas en el prototipo, durante las últimas pruebas desarrolladas en la Facultad de Enfermería de la UPCH, de ello se concluye que:
Figura 2. Primera experimentación con usuarios en el Laboratorio de Innovación Tecnológica de la Facultad de Enfermería de la UPCH
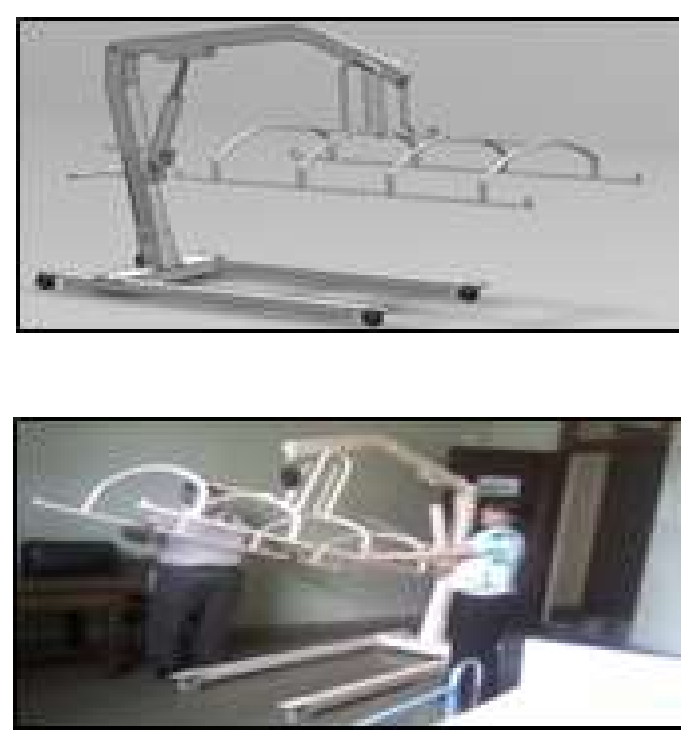
Se integrará cuatro barras (paralelogramo) al mecanismo ya existente del prototipo de grúa, a fin de evitar el balanceo de la camilla con el paciente. Como parte de esta integración, se modificará la canastilla para su instalación en la barra de acople.

Se integrará una balanza al mecanismo del prototipo grúa.
3. Se presentó el prototipo integrando la camilla recta, la balanza electrónica y el sistema de comunicación vía bluetooth, el manejo y el uso de todas las funcionalidades (lectura de pesos, uso y manejo del sistema integrado) fueron explicados al personal de la Facultad de Enfermería de la UPCH, para su posterior validación con los pacientes (Figura 5).

Figura 3. Prototipo integrado por la balanza electrónica
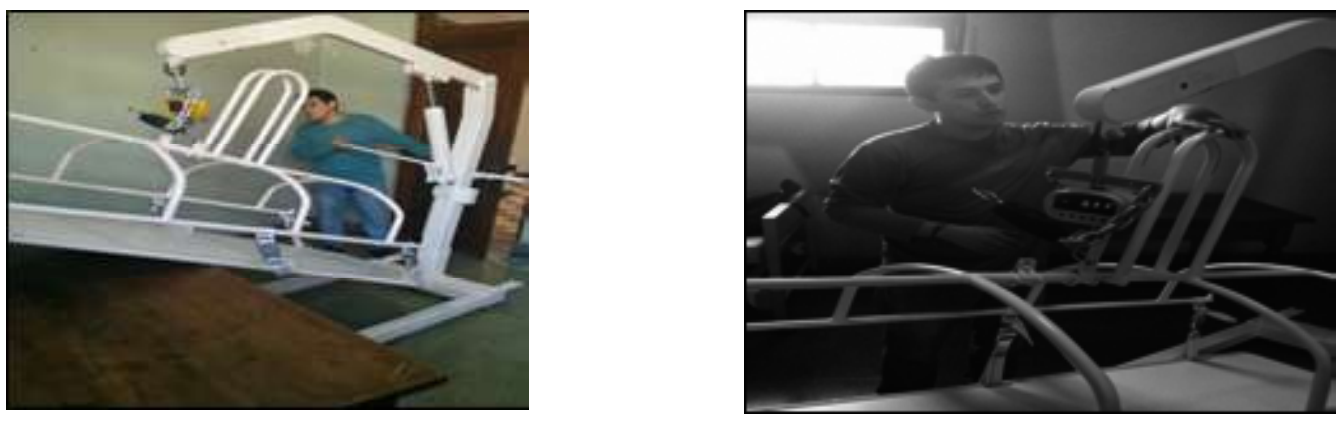

Figura 4. Planteamiento en pizarra de las posibles correcciones al prototipo
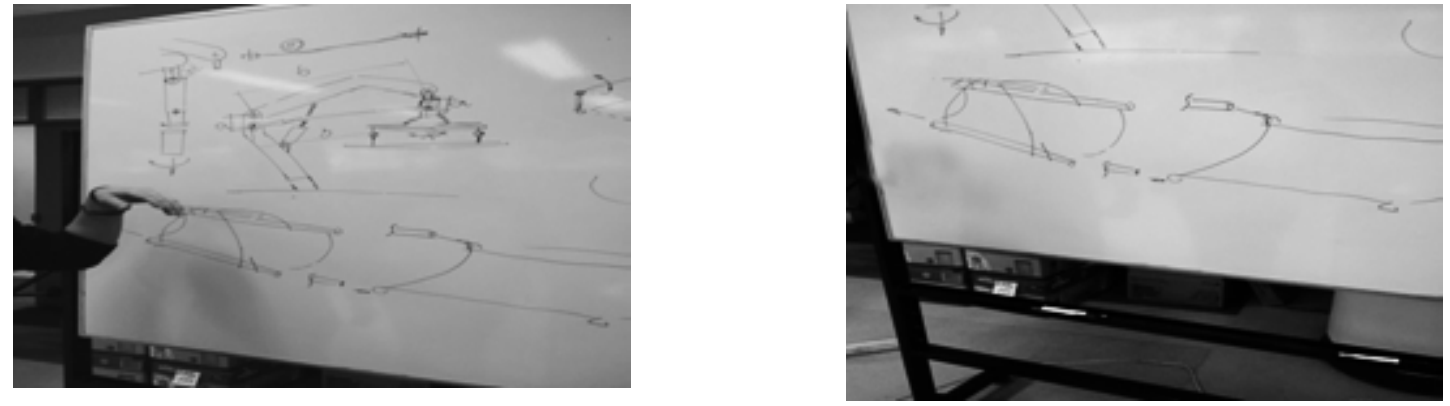

Figura 5. Personal de la FAENF probando el prototipo
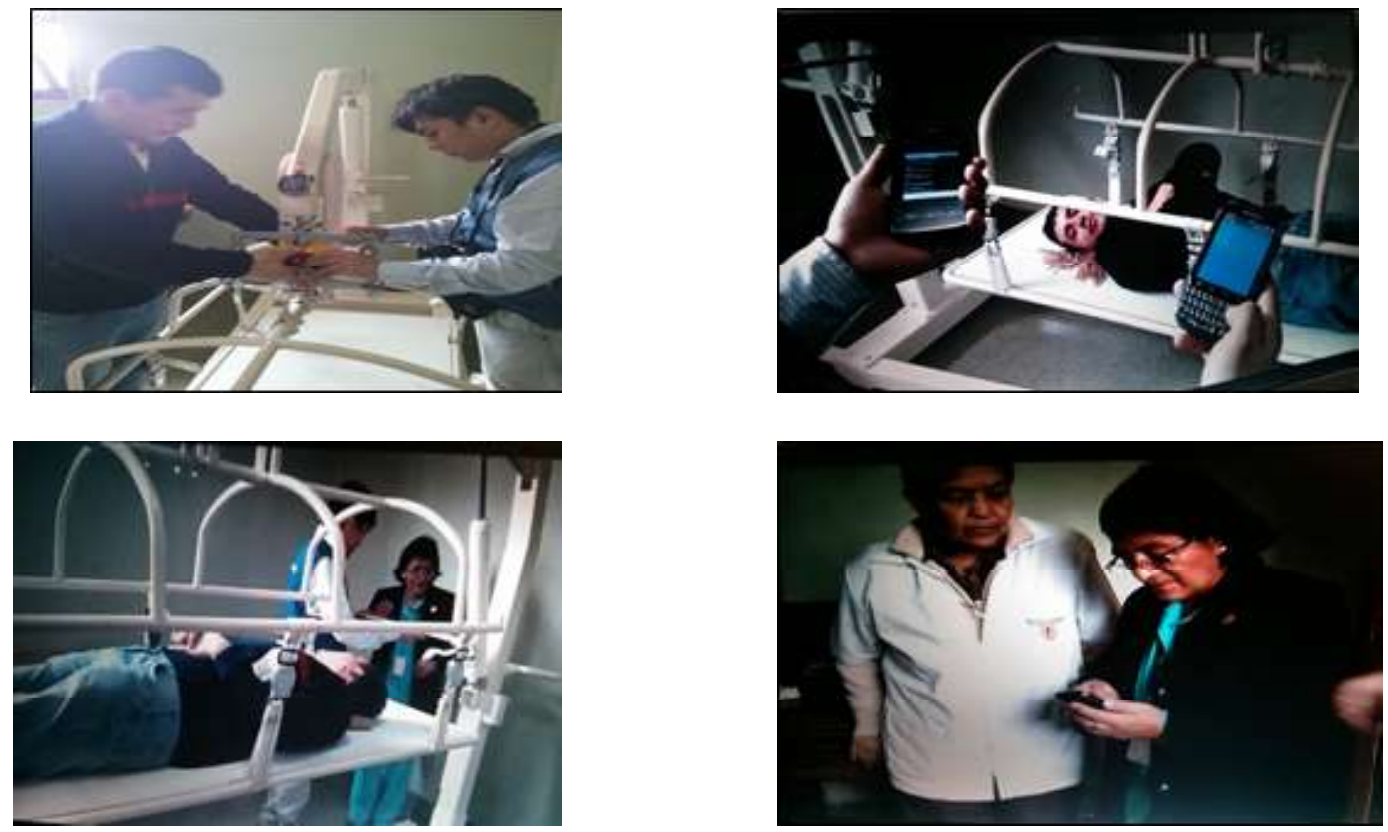
MEJORAS PROPUESTAS POR EL EQUIPO DE INVESTIGACIÓN DE LA UNI

4. Después de la reunión del equipo se diseña un modelo actualizado del prototipo (el cual no se concretó por la falta de medios financieros, materiales y humanos), y se plasma como se muestra en las siguientes imágenes:

Figura 6. Modelo actualizado del prototipo
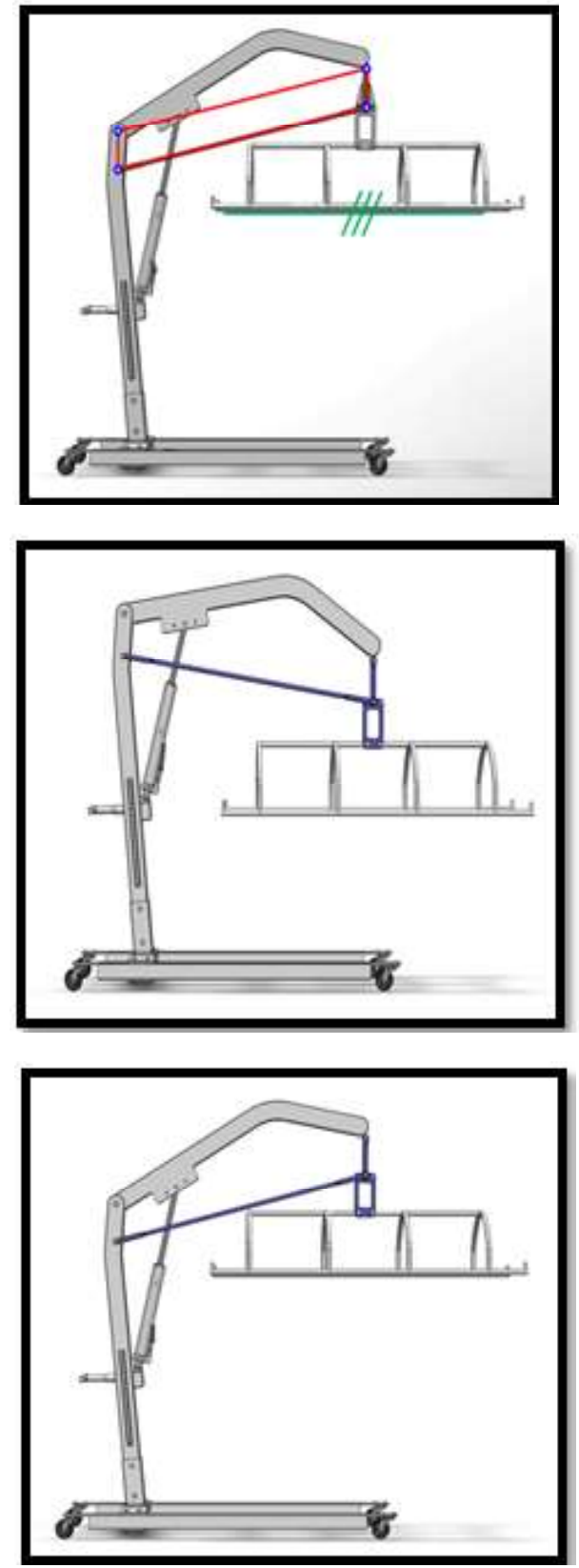

VALIDACIÓN INTERNA DE PARTE DEL EQUIPO DE INVESTIGACIÓN DE LA UPCH

Para evaluar la confiabilidad de las mediciones realizadas con el uso del prototipo, se pesó a diez personas y se comparó dichas mediciones con un gold estándar, en este caso, una balanza de pie, previamente calibrada. No se
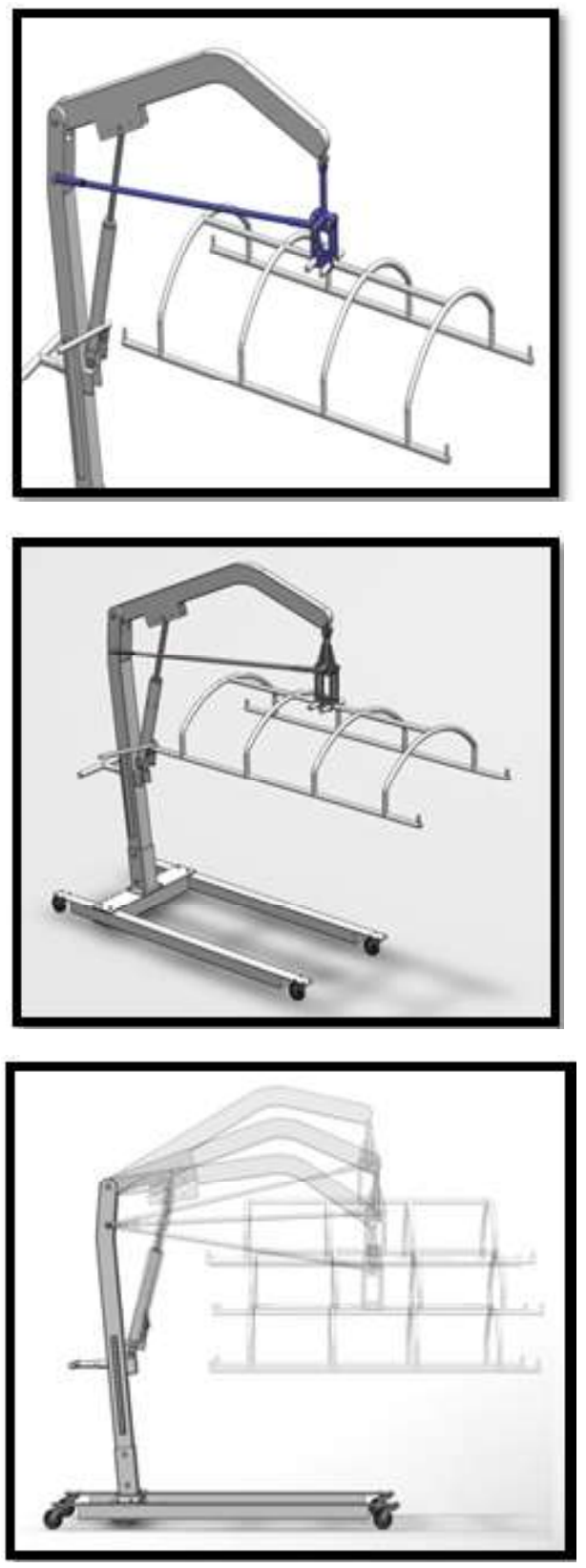

hizo una mayor cantidad de pesaje, dado que el equipo mostraba inestabilidad al elevar a los sujetos, pues se corría el riesgo de lesión o caída.

En la tabla 1, en el resultado se observa que no existe diferencia significativa ( $p>0,05)$, lo que implica que no hay diferencia al comparar las medias de los pesos obtenidos 
entre ambas balanzas. Sin embargo, al analizar la diferencia mínima entre los pesos obtenidos, esta fue de $100 \mathrm{~g}$ y la diferencia máxima de $24 \mathrm{k}$, con una diferencia promedio de $9 \mathrm{~kg} \pm 10 \mathrm{~kg}$. En el caso de precisión en el peso, es una diferencia muy importante, más aun si este peso debe determinar la dosis de medicamentos o la reposición de líquidos en un balance hídrico.

El equipo evaluador notó en esta evaluación que para registrar el peso de cada persona debió esperarse un promedio de un minuto, hasta que el registro de la pantalla dejara de oscilar. Adicionalmente, al cabo de tres o cuatro mediciones seguidas, la diferencia del peso de la balanza se iba incrementando de modo notable, hasta llegar a los 20 kilos en promedio. Frente a dicha situación, se decidió evaluar el peso de los objetos inertes, de esta manera, la balanza no oscilaba mucho y la precisión en el peso resultaba mejor, tal como lo muestra la siguiente tabla.
En la tabla 2, en el resultado se puede observar que no hay significancia estadística ( $p>0,05)$, esto es, que no existe diferencia significativa al comparar las medias de los pesos obtenidos con ambos equipos. Sin embargo, el promedio de la diferencia de pesos entre una balanza y otra fue de solo $760 \mathrm{~g}$, teniendo una diferencia máxima al pesar los objetos de 2890 gramos, cerca de tres kilos. El tiempo empleado en registrar el peso en la balanza fue de $10 \mathrm{a}$ $15 \mathrm{~s}$. El registro tuvo menos oscilación que al pesar personas.

Se concluye que el proceso de pesaje empleado para usar el prototipo de balanza para pacientes postrados no es válido, debido a que el tamaño del equipo no se adapta al de la cama convencional hospitalaria. El valor otorgado como peso por el prototipo de balanza para pacientes postrados es considerado no confiable, dado que la diferencia de pesos en promedio con el gold estándar fue de aproximadamente $10 \mathrm{k}$.

Tabla 1. Valores obtenidos al usar cada balanza Comparación de los pesos de personas obtenidos al usar la balanza convencional y el prototipo (valores en gramos)

\begin{tabular}{|c|c|c|c|c|c|c|c|c|}
\hline $\mathrm{n}=10$ & Media & Dess. & $\begin{array}{c}\text { Diferencia } \\
\text { mínima }\end{array}$ & $\begin{array}{c}\text { Diferencia } \\
\text { máxima }\end{array}$ & $\begin{array}{l}\text { Diferencia } \\
\text { entre los } \\
\text { pesos de los } \\
\text { equipos }\end{array}$ & $\begin{array}{c}\text { DSde la } \\
\text { diferencia } \\
\text { entre los } \\
\text { pesos de los } \\
\text { equipos }\end{array}$ & $t$ & $\begin{array}{c}\text { Sig. } \\
\text { estadística }\end{array}$ \\
\hline $\begin{array}{l}\text { Balanza } \\
\text { convencional } \\
\text { Prototipo }\end{array}$ & $\begin{array}{l}69106 \\
78982\end{array}$ & $\begin{array}{l}14111 \\
16893\end{array}$ & 100 & 24000 & 9,876 & 10447 & $-1,4$ & $0,17^{*}$ \\
\hline
\end{tabular}

Tabla 2. Comparación de los pesos de objetos obtenidos al usar la balanza convencional y el prototipo (valores en gramos)

\begin{tabular}{lcccccccc}
\hline $\mathrm{n}=30$ & Media & Desviación & $\begin{array}{c}\text { Diferencia } \\
\text { minima }\end{array}$ & $\begin{array}{c}\text { Diferencia } \\
\text { máxima }\end{array}$ & $\begin{array}{c}\text { Diferencia } \\
\text { entrelos } \\
\text { pesos de } \\
\text { los equipos } \begin{array}{c}\text { DS dela } \\
\text { diferencia } \\
\text { entrelos } \\
\text { pesos delos } \\
\text { equipos }\end{array}\end{array}$ & $\begin{array}{c}\text { Sig. } \\
\text { estadistica }\end{array}$ \\
\hline $\begin{array}{l}\text { Balanza } \\
\text { convencional } \\
\text { Prototipo }\end{array}$ & 3420 & 1760 & 0 & 2890 & 760 & 1170 & 1,81 & $0,075^{*}$ \\
\hline
\end{tabular}

*No significativo

\section{REFERENCIAS BIBLIOGRÁFICAS}

1. Monckeberg F. La revolución de la bioingeniería (Internet). Mediterráneo, 1988. (Citado el 31 de Oct. 2012).

2. Poblet JM. Introducción a la Bioingeniería. Marcombo, 1988. (Citado el 29 de Oct. 2012)
3. Rodríguez E. Enseñanza de la Ingeniería Biomédica en Cuba. Centro, 2003: 602:604.

4. Swan GW. Optimal control applications in biomedical engineering. Opt. Control Appls. Methods 1980; 2:11334. Disponible en: http://www.readcube.com/articles/ $10.1002 \% 2$ Foca.4660020402?r3_referer $=$ wol\& tracking_action $=$ preview_click\&show_checkout $=1$. 
5. Dirección General de Epidemiología. Análisis de la Situación de salud del Perú. Ministerio de salud. Lima; 2010. Disponible en: http://www.dge.gob.pe/ publicaciones/pub_asis/asis25.pdf.

6. Resultado de encuestas aplicadas en la Facultad de Enfermería de la Universidad Peruana Cayetano Heredia, octubre de 2012 (uso interno).

7. Básculas médicas Seca: tallimetro de aluminio para básculas Seca. Disponible en: http://www.solo stocks.com/venta-productos/mobiliario/otros/ basculas-medicas-seca-tallimetro-de-aluminio-parabasculas-seca-644-645-5601963.

8. TYP Instrumentos [Internet]. Balanzas para transacciones comerciales, de farmacia, uso médico y servicio técnico. Disponible en: http://www.typinstrumentos.com/attachments/184_Lista $\%$ 20de $\% 20$ precios \%20Balanzas\%20TYP-Instrumentos.\% 202011.pdf

9. Ortiz SP, Manrique HH, Solís VJ, Candioti HM, el al. «Prevalencia de desnutrición en los servicios de hospitalización de medicina». Revista de la Sociedad peruana de Medicina Interna. 2007; 20(1): 16-20.

10. Oliveros Pantoja I, Hernández Donado R. Bioingeniería: Solución a problemas de las ciencias biológicas y médicas apoyados en la Ingeniería. Ingeniería \& Desarrollo. Universidad del Norte. 5: 105-111, 1999. Disponible en: http://rcientifi cas.uninorte.edu.co/index.php/ingenieria/article/view/ 2214/1435.

11. Blanco G, Gariboto M, Gonzáles C, Lanetta B. Instrumentos de pesaje para personas amputadas o postradas. Revista electrónica de Ciencias Aplicadas al Deporte. 2012; 5 (16). Disponible en: http:// romerobrest.edu.ar/ojs/index.php/ReCAD/article/ view/10.

12. Weisz RM, Carrere LC, Añino M M. Modelización, simulación y práctica experimental en el ciclo básico de bioingeniería: una propuesta didáctica. Revista
Educación en Ingeniería. 2012; 7 (14): 58-70. Disponible en: http://www.educacioneningenieria.org/ index.php/edi/article/view/246/154

13. Valentinuzzi M E. Introducción a la bioingeniería [Internet].Barcelona: Marcombo; 1988. [citado $13 \mathrm{Set}$ 2013].URL Disponible en: https://books.goo gle.com.pe/books?id=aqcaSGADoo4C\&pg $=$ PP13\& lpg $=\mathrm{PP} 13 \& \mathrm{dq}=$ bioingenier

14. Yabar L, Condor C, García D, Villavicencio E. La ingeniería Biomédica en el Perú: retrospectiva y situación actual. IV Latin American Congress on Biomedical Engineering 2007, Bioengineering Solutions for Latin America Health IFMBE Proceedings 2008; $18: 820-824$. Disponible en: http://link.springer.com/ chapter/10.1007/978-3-540-74471-9_190\#page-1.

15. Neira Meloni RE, et al. Construcción de una Camilla automatizada para bipedestación. XVIII Congreso Argentino de Bioingeniería SABI-2011, Mar de Plata, 28 al 30 de sePtiembre de 2011.

\section{Correspondencia}

Rosa Vaiz Bonifaz

Universidad Peruana Cayetano Heredia

Dirección: Av. Honorio Delgado 430, Urb. Ingeniería, Lima - 31

Correo electrónico: rosav@upch.pe

Forma de citar este artículo: Ortega-Loayza D; Cisneros-Morales C; Tabuchi-Yaqui T; Arteaga-Chávez L; Mayorca-Carmelo C; Vaiz-Bonifaz R; Bolaños Hil H; Salazar-García M; Farro-Peña G. Prototipo de bioingenieria para el traslado y pesaje de pacientes hospitalizados con sobrepeso o movilidad limitada. Rev enferm Herediana. 2014;7(2):79-86. 